\title{
Child Behavior Checklist: Cuestionario de conductas socio-afectivas en una población preescolar de Cienfuegos, Cuba.
}

\author{
Child Behavior Checklist: Socio-affective behavior questionnaire in a preschool population in Cienfuegos, \\ Cuba.
}

\author{
Yaser Ramírez-Benítez 1,a, Rodneys Mauricio Jiménez-Morales ${ }^{2, b}$. Bárbara Bermúdez-Monteagudo 1,c
}

\section{RESUMEN}

La evaluación del desarrollo afectivo en la infancia preescolar es una necesidad en Cuba debido a la baja tasa de mortalidad infantil que puede tener en adelante, como consecuencia, niños con diversos riesgos de salud; por otro lado y en el caso concreto de problemas afectivos, existen pocas pruebas válidas para su detección temprana. Objetivo: Comprobar la validez del cuestionario Child Behavior Checklist en la provincia de Cienfuegos, y caracterizar el desarrollo afectivo en una muestra de niños entre 4 y 6 años de edad con necesidades educativas especiales. Material y Métodos: Se utilizaron dos muestras, N1 (320 niños) como grupo de referencia para obtener percentiles y realizar la validación, y N2 (60 niños) como muestra con alteraciones de desarrollo, para validar los percentiles y caracterizar a potenciales pacientes. Resultados: Se encontraron adecuados valores de confiabilidad (alfa cuando el ítem se elimina entre 0.84 y 0.85 ). La validez de contenido indicó un adecuado ajuste cultural del cuestionario en la población cubana y un 96,7\% de los ítems con buenos y/o aceptables niveles de discriminación. En N2 se encontraron varios niños con alteraciones emocionales y conductuales $(88,3 \%$, tres cuartas partes de los cuales eran varones): Las alteraciones fueron manifiestas en las sub-escalas: Introversión, Atención, Sueño y Ansiedad. Conclusiones: El cuestionario Child Behavior Checklist (versión para padres) es una herramienta valiosa para profesionales cubanos de salud mental, pues permite clasificar expresiones afectivas negativas de tipo exteriorizante o interiorizante en la infancia preescolar. Este tipo de estudio debe realizarse en una muestra de referencia nacional a fin de revisar los percentiles y comprobar o confirmar los hallazgos de validez y confiabilidad.

PALABRAS CLAVE: Emociones, edad preescolar, ansiedad, atención, sueño.

\section{SUMMARY}

The evaluation of socio-affective development in the child population is perceived as necessity in Cuba's clinical and educational actions due to the low mortality levels that can result in the presence of several health risks among children; on the other hand, and in the specific case of affective problems, there are few valid and reliable tests for their early detection. Objective: To validate the Child Behavior Checklist questionnaire for a preschool population sample (4-6 years) with special needs, in the Cienfuegos province of Cuba. Material and Methods: Two samples, N1 (320 children) as a reference group to obtain percentiles and perform content validation, expert and internal

Departamento de Logopedia y Educación especial, Universidad de Cienfuegos. Cienfuegos, Cuba.

Centro de Estudio de Ciencias de la Educación, Universidad de Santi Spíritus. Sancti Spíritus, Cuba.

M.Sc. Neurociencias cognitivas. Doctorante Ciencias Pedagógicas.

b PhD. Ciencias Psicológicas.

c Docente e investigador. PhD. Ciencias Pedagógicas 
consistency, and N2 (60 children) with developmental alterations to validate the percentiles and characterize future potential patients. Results: Adequate reliability values (alpha when the item is removed between .84 and .85 ) were found. The validity of the content indicated an adequate cultural adjustment of the questionnaire in the Cuban population, and $96.7 \%$ of the items with good and/or acceptable discrimination. In N2, $88.3 \%$ of the probands (and three fourths of them, boys) were found carrying emotional and behavioral disorders. The abnormal findings occurred, particularly, in the Introversion, Attention, Sleep and Anxiety sub-scales. Conclusions: The Child Behavior Checklist questionnaire (version for parents) is a valuable alternative for Cuban mental health professionals, as it allows classifying negative affective expressions of externalizing or internalizing type in preschool children. Studies in national reference samples must be conducted in order to test or confirm these validity and reliability findings.

KEYWORDS: Emotions, preschool age, anxiety, attention, sleep.

\section{INTRODUCCIÓN}

El desarrollo socio afectivo en la población infantil es un tema controvertido dentro de las ciencias del comportamiento debido a la complejidad para evaluar esta área del desarrollo, tanto en la Psicología, la Neurología, la Psiquiatría y en las diferentes disciplinas de la Educación.

El debate del tema se ha centrado en tres posiciones críticas: 1) poco consenso entre profesionales para determinar comportamiento típicos o patológicos en la población infantil, 2) poca confiabilidad de la información que los padres brindan sobre el comportamiento de sus hijos y, 3) insuficientes pruebas para evaluar el desarrollo socio afecto en población infantil.

Los sentimientos y emociones en el niño tienen una expresión similar a un adulto, sin embargo, la duración, la intensidad y la intensión pueden ser diferentes. El niño puede pasar de un estado de ánimo a otro con facilidad, puede estar todo el tiempo que quiere riendo o llorando sin saber cuándo acabar, y también puede usar los sentimientos y el estado de ánimo para obtener lo que desee sin importar las consecuencias de las acciones.

Estas características son conocidas por los adultos, pues la relación niño - adulto es una adecuada forma de conocer y estimular el desarrollo socio afectivo en la población infantil. Sin embargo, resulta complejo identificar si el niño está mintiendo o si su comportamiento es expresión de una enfermedad mental o neurológica.

Una manera adecuada de establecer diferencias es observando la frecuencia en que se expresan los estados de ánimo o una actitud negativa hacia una persona, un animal o en una situación $(1,2,3)$; en otras palabras, un niño que muestra agresividad en el aula, en su casa y en el juego con otros compañeros debe ser valorado por un especialista. No obstante, las observaciones no son tan simples, pues las expresiones no son tan claras y en ocasiones suele estar ocultas a los ojos del adulto, ya que el niño aprende a conocer las conductas negativas por los regaños y los castigos. De 0 a 6 años, esta situación es mucho más compleja, pues las expresiones emocionales negativa de tipo patológicas son frecuentes tanto en el niño enfermo como en el niño con desarrollo típico, por tanto resulta complejo diferenciar cuándo es hiperactivo, agresivo, desafiante, ansioso o deprimido. En la infancia preescolar, es típico encontrar estas manifestaciones de naturaleza conductual y socio afectiva, sin embargo detectar expresiones frecuentes en esta edades es una oportunidad para frenar o disminuir conductas negativas que tendrán mayores consecuencias en la etapa escolar, tanto para la formación de la personalidad del niño, como para su aprendizaje.

Otro punto de interés es la confiabilidad de la información que brindan los padres, pues en algunas familias la entrevista es suficiente para detectar las expresiones emocionales negativas del niño preescolar, aunque en la mayoría de los informantes (los padres y educadores) es necesario usar varias técnicas o métodos, tales como el cuestionario, la observación del niño en el grupo y la entrevista. Desde esta exigencia metodológica, en la actuación profesional es complejo encontrar pruebas o cuestionarios para valorar el desarrollo socio afectivo en la infancia preescolar, especialmente pruebas que expresen resultados válidos, confiables y con normativas para clasificar.

Un valioso cuestionario es el Child Behavior Checklist versión para padres $(\mathrm{CBCL})$, el cual permite detectar conductas negativas del niño en diferentes espacios de actuación (4). Otro cuestionario con similares características es el Behavior Assessment System 
for Children (BASC) (5), sin embargo, al parecer, el CBCL ha tenido una apropiada aceptación en la comunidad científica latinoamericana, pues en la literatura se han publicados varios estudios de validación y estandarización en diferentes países de habla hispana, tales como en Chile (6), en Venezuela (7), en Argentina (8), en México (9) lo cual indica su alta utilidad práctica tanto en los instituciones de salud, como en las educacionales. No obstante, en Cuba no se han reportado estudios de validez o de estandarización del cuestionario, incluso pocos estudios lo han utilizado.

En Cuba, resulta necesario contar con un cuestionario o pruebas sobre el desarrollo emocional de los niños preescolar debido a tres razones: 1) tasas de mortalidad cada vez más bajas (índice entre 3 y 4 por 1000 nacimientos), por tanto puede aparecer más niños con prematuridad, bajo peso al nacer, con desordenes metabólicos, con antecedentes de hipoxia, los cuales pueden expresar diversas conducta negativas en la primera infancia (agresividad, hiperactividad, desórdenes del sueño, de la alimentación, enfermedades auto-inmunes que generen malestares físicos y emocionales permanente o intermitente en varios escenario del desarrollo), 2) aumento de niños preescolar con alteraciones cognitivas en la provincia de Cienfuegos, tanto en instituciones clínicas como educativas (10), lo cual debe ser revisado con una prueba que emita resultados válidos y confiables, pues acompañado de los problemas cognitivos aparecen las alteraciones socio emocionales, y 3 ) en la infancia preescolar ocurren los principales acelerones biológicos, psicológicos y educativos para establecerse el desarrollo socio afectivo del niños, por tanto, si el niño con alteraciones no recibe orientaciones puntuales y oportunas puede generar conductas negativas a largo plazo. Por tomar un ejemplo, la agresividad sin una educación permanente puede crear rechazo del niño en varios escenarios escolares y públicos.

Teniendo en cuenta la importancia de la detección temprana de las conductas y expresiones emocionales negativas en la infancia preescolar, y la utilidad práctica del cuestionario para padres CBCL, la presente investigación tiene como objetivos: 1) Validar y adaptar el cuestionario Child Behavior Checklist para niños preescolar entre 4 y 6 años en la provincia de Cienfuegos, y 2) Caracterizar el desarrollo socio afecto en una muestra de niños entre 4 y 6 años en la provincia de Cienfuegos con necesidades educativas especiales.

\section{MATERIAL Y MÉTODOS}

Para validar el cuestionario de conducta se seleccionaron 380 niños preescolares con edades comprendidas entre 4 y 6 años de ambos sexos, provenientes de varias instituciones educativas en la provincia de Cienfuegos.

La selección de la cantidad de niños, las instituciones educativas y la provincia se hizo considerando las intenciones de los investigadores: 1) conocer el funcionamiento inicial del cuestionario para identificar ítems o preguntas con un mal funcionamiento o con baja compresión, por eso se seleccionó como mínimo 100 niños por grupos de edades, 2) en las instituciones se seleccionaron los niños por años de vida: 4to año de vida (entre 46 y 48 meses), 5to año de vida (entre 58 y 60 meses) y 6to año de vida (entre 70 y 78 meses), 3) se aplicó en una provincia donde fue adaptado el cuestionario, para luego realizar un estudio con una muestra representativa del país, y 4) se seleccionaron los niños de los Círculos infantiles y Escuelas primarias regulares por ser instituciones educativas de permanente estimulación.

La muestra total se dividió en dos sub-muestras independientes: N1 (320 niños, 101 niños con 4 años, 105 niños con 5 años y 114 niños con 6 años) y N2 (60 niños, 20 con 4 años, 20 con 5 años y 20 con 6 años). La muestra N1 constituyó el grupo de referencia y por consiguiente se incluyeron niños con desarrollo mental y socio afectivo típico para su edad cronológica. La selección se realizó considerando el criterio de la educadora, la Psicopedagoga y/o de la Logopeda de la institución educativa. Con esta N1 (320 niños) se calculó los percentiles por edades para identificar el perfil socio afectivo de tipo Internalizante (niños con una ligera o marcada inclinación hacia la ansiedad, la depresión y la introversión) y de tipo Externalizante (niños con una ligera o marcada inclinación hacia la agresividad, la hiperactividad y los problemas de atención).

La muestra N2 se seleccionó de 21 instituciones educativas (15 círculos infantiles y 6 escuelas primarias) para validar los percentiles obtenidos. La selección de los niños se hizo considerando un criterio de inclusión: niños con posible trastorno socio afectivo o con trastornos de adaptación tratados por especialistas del círculo (Psicopedagogía o Logopedia) y/o por el Centro de Diagnóstico y Orientación (CDO). La Dirección Provincial del Ministerio de Educación en la provincia de Cienfuegos autorizó la entrada a 
las instituciones educativas. Asimismo, se solicitó el consentimiento informado a los padres de los niños seleccionados en el estudio. La descripción de la muestra N1 y N2 se observa en la tabla 1.

\section{Instrumento}

Cuestionario de Conducta para preescolares (Child Behavior Checklist): El Child Behavior Checklist (CBCL) versión para padres es un instrumento que contiene 100 ítems para valorar el desarrollo socio afectivo de niños entre $1 \frac{1}{2}$ y 5 años (4). Los ítems agrupan 8 sub-escalas que permiten valorar de manera empírica la conducta del niño y su desarrollo socio afectivo. Sub-escala 1: Emocionalmente Reactivo (ítems 21, 46, 51, 79, 82, 83, 92, 97 y 99). Sub-escala 2: Ansiedad - Depresivo (ítems 10, 33, 37, 43, 47, 68, 87 y 90). Sub-escala 3: Quejas somáticas (ítems $1,7,12,19,24,39,45,52,78,86$ y 93). Sub- escala 4: Introvertido (ítems 2, 4, 23, 62, 67, 70, 71 y 98). Sub-escala 5: Problemas en el sueño (ítems 22, 38, 48, 64, 74, 84 y 94). Sub-escala 6: Problemas de atención (ítems 5, 6, 56, 59, 95). Sub-escala 7: Conducta agresiva (ítems $8,15,16,18,20,27,29,35,40,42,44$, $53,58,66,69,81,85,88$ y 96). Sub- escala 8 : Otros problemas (ítems 3, 9, 11, 13, 14, 17, 25, 26, 28, 30, $31,32,34,36,41,49,50,54,55,57,60,61,63,65,72$, $73,75,76,77,80,89,91$ y 100). Con 7 sub-escalas se conforman tres escalas generales: Internalizante (EI) (suma de las puntuaciones de las Sub-escalas 1, 2, 3 y 4), Externalizante (EE) (suma de las puntuaciones de las Sub-escalas 6 y 7) y Problemas generales (PG) (suma de las puntuaciones de las Sub-escalas 5 y 8). Las tres escalas (EI, EE y PG) y las sub-escalas generales presentan percentiles para clasificar a los niños dentro de tres rasgos: Típico (sin alteraciones), Preclínico (presencia de algunas alteraciones) y Clínico (con alteraciones).

\section{Análisis estadísticos}

Estadística descriptiva: se aplicó la prueba t para muestras relacionadas con la intención de comparar el rendimiento de las sub-muestras N1 y N2. Con igual propósito se aplicó la $d$ de Cohen para conocer el tamaño del efecto entre N1 y N2. Los valores de la $d$ de Cohen se calificaron según Cohen (1988): $\leq 0,20$ efecto pequeño, $\geq 0,50$ efecto mediano $\mathrm{y} \geq 0,80$ efecto grande. Se aplicó un análisis de percentiles para clasificar los niños con rasgos clínicos y preclínicos. Validez de contenido: Se realizó un análisis de ítems con ambas sub-muestras (N1 y N2) para obtener y analizar en conjunto con el análisis de factores la validez de contenido. El análisis de ítems se realizó considerando los valores del índice de discriminación y el índice de dificultad de los ítems. El índice de discriminación se clasificó según los criterios de Backhoff, Larrazolo \& Rosas (11): Ítem con Buena Discriminación $(\geq 0,20)$, Ítem con Aceptables Discriminación $(0,10$ y 0,19$)$, los cual debe ser revisado en próximo estudios, y Ítem con Mala Discriminación $(<0,10)$, los cual deben ser rechazado o modificados. El índice de dificultad se clasificó según Crocker y Algina (12) en: Dificultad Baja ( $\geq 0.80$ ); Dificultad Media (entre 0.79 y 0.50 ) y Dificultad Alta $(<0.50)$. Se realizó, un análisis de expertos para revisar la compresión del cuestionario y adaptar algunos vocablos al contexto cultural cubano.

Validez de estructura interna: Se realizó un análisis confirmatorio de factores para obtener la validez de estructura interna. Para realizar este procedimiento primero se tuvo que justificar si era pertinente a través de la prueba Kaiser-Meyer-Olkin (KMO). La prueba KMO cuando alcanza valores $\geq 0,75$ la idea de realizar un análisis factorial es buena, si 0,75 $>\mathrm{KMO} \geq 0,5$ la idea es aceptable y si $\mathrm{KMO}<0,5$ es inaceptable (13).

Confiabilidad: se determinó a través de dos procedimientos: (1) Alfa cuando el ítem es eliminado y (2) Correlación ítem-total con el objetivo de descartar ítems que no contribuyen de manera significativa a la varianza de la prueba.

\section{RESULTADOS}

\section{Estadística descriptiva}

La prueba $t$ de Student indicó diferencias significativas entre la muestra N1 y N2, en específico fue más significativo en las sub-escalas: Emocionalmente reactivo $(5,712 ; \mathrm{p} \leq 0,000)$, Ansiedad $(8,445 ; \mathrm{p} \leq 0,000)$, Introvertido $(8,821 ; \mathrm{p} \leq 0,000)$, Sueño $(4,320 ; \mathrm{p} \leq 0,000)$, Atención $(7,446 ; \mathrm{p} \leq 0,000)$, Agresivo $(3,652 ; \mathrm{p} \leq 0,000)$ e Internalizante $(7,132$; $\mathrm{p} \leq 0,000)$. El análisis con la $d$ de Cohen indicó una diferencia significativa (efecto grande) entre N1 y N2, específicamente con un efecto grande en las variables Introvertido $(d=1,18)$, Internalizante $(d=1,13)$, Atención $(d=1,07)$, Agresivo $(d=0,90)$ y Ansiedad $(d=0,81)$, mientras mostró un efecto mediano en las variables Emocionalmente reactivo $(d=0,69)$ y Problema de Sueño $(d=0,80)$, tal y como se muestra en la tabla 2.

El análisis de percentiles indicó una adecuada distribución de los datos como se muestra en la tabla 3 , 
Tabla 1. Descripción de la muestra N1 y N2.

\begin{tabular}{|c|c|c|c|c|c|c|c|c|c|c|}
\hline \multirow{3}{*}{ Grado } & \multicolumn{5}{|c|}{ N1 } & \multicolumn{5}{|c|}{$\mathrm{N} 2$} \\
\hline & \multirow[t]{2}{*}{ Total } & \multicolumn{2}{|c|}{ Género } & \multicolumn{2}{|c|}{ Edad } & \multirow[t]{2}{*}{ Total } & \multicolumn{2}{|c|}{ Género } & \multicolumn{2}{|l|}{ Edad } \\
\hline & & $\mathbf{F}$ & $\mathbf{M}$ & Media & $\mathbf{D E}$ & & $\mathbf{F}$ & $\mathbf{M}$ & Media & DE \\
\hline 4to año de vida & 101 & 45 & 56 & 4,3 & 0,29 & 20 & 9 & 11 & 4,5 & 0,34 \\
\hline 5to año de vida & 105 & 49 & 56 & 5,2 & 0,11 & 20 & 8 & 12 & 5,3 & 0,29 \\
\hline 6to año de vida & 114 & 55 & 59 & 6,5 & 0,20 & 20 & 7 & 13 & 6,4 & 0,26 \\
\hline Total & 320 & 152 & 168 & 5,3 & 0,25 & 60 & 24 & 36 & 5,4 & 0,28 \\
\hline
\end{tabular}

Nota: DE (desviación estándar), F (Femenina), M (Masculino)

Tabla 2. Puntuaciones en el cuestionario de las sub-muestra N1 y N2.

\begin{tabular}{lrrrrrrc}
\hline \multicolumn{1}{c}{ Variables } & \multicolumn{2}{c}{ N1 } & \multicolumn{2}{c}{ N2 } & \multicolumn{3}{c}{ Prueba t } \\
& $\mathbf{M}$ & $\mathbf{D E}$ & $\mathbf{M}$ & $\mathbf{D E}$ & $\mathbf{t}$ & Sign. & $\mathbf{d}$ \\
\hline Emocional & 3,00 & 1,43 & 4,03 & 1,20 & 5,712 &, 000 & 0,71 \\
Ansiedad & 2,31 & 1,11 & 3,30 & 0,84 & 8,445 &, 000 & 0,81 \\
Somático & 2,00 & 1,30 & 2,35 & 1,24 & 1,101 &, 910 & 0,20 \\
Introvertido & 1,05 & 0,93 & 2,70 & 1,56 & 8,821 &, 000 & 1,81 \\
Sueño & 2,52 & 1,31 & 3,66 & 0,98 & 4,320 &, 000 & 0,83 \\
Atención & 2,11 & 1,28 & 3,45 & 1,15 & 7,446 &, 000 & 1,07 \\
Agresivo & 5,51 & 3,24 & 2,76 & 2,59 & 3,652 &, 001 & 0,90 \\
Otros problemas & 3,61 & 3,45 & 3,36 & 1,05 & 1,481 &, 304 & 0,08 \\
Internalizante & 8,21 & 4,22 & 12,38 & 2,64 & 7,132 &, 000 & 1,13 \\
Externalizante & 7,50 & 4,36 & 6,21 & 2,66 & 2,014 &, 981 & 0,44 \\
Prob. Generales & 6,30 & 4,28 & 7,03 & 1,47 & 1,345 &, 687 & 0,19 \\
\hline Nota: En la primera columna las sub-escalas del cuestionario. En la segunda columna los \\
valores descriptivos de la sub-muestra N1 (Media; Desviación Estándar; RP, Cantidad de \\
niños con Rasgos Preclínico y RC, Cantidad de Niños con Rasgos Clínicos). En la tercera \\
columna los valores descriptivo de la sub-muestra N2. En la cuarta columna los valores de \\
la prueba t entre las dos sub-muestra y los valores de la $d$ de Cohen. \\
\end{tabular}

lo cual permitió realizar una aceptada caracterización de la muestra N2.

\section{Caracterización socio afectiva de la muestra N2}

En la muestra N2, el cuestionario mostró que la mayoría de los niños $(88,3 \%)$ presentaron alteraciones socio afectivo, en especial en las subescalas de Atención, Ansiedad, Problemas de sueños e Introvertido. Con igual interés, el análisis por sexo indicó que el $75 \%$ de los niños con rasgos preclínicos y clínicos son varones, mientras que un $15 \%$ fueron hembras. Las principales alteraciones estuvieron en las sub-escalas Introvertido (de 25 niños 15 varones), Atención (de 22 niños 14 varones), Ansiedad (de 14 niños 9 varones) y Problemas del sueño (de 13 niños 7 varones).
A los 4 años, la mayor cantidad de niños con rasgos preclínicos se dieron en las sub-escalas de Introvertido (9 niños), Atención (8 niños) y Ansiedad (6 niños) (figura 1), mientras que la mayoría de los niños con rasgos clínicos fueron en la Emocional (2 niños), Ansiedad (2 niños) y Agresivo (2 niños) (figura 2).

A los 5 años, la mayor cantidad de niños con rasgos preclínicos se presentaron en las sub-escalas de Problemas de Sueño (11 niños), Atención (9 niños), Ansiedad (6 niños) e Introvertido (6 niños) (figura 1), mientras que la mayoría de los niños con rasgos clínicos se presentaron en las sub-escalas de Problemas de Sueño (2 niños) e Introvertido (2 niños) (figura 2). A los 6 años, la mayor cantidad de niños con rasgos preclínicos se presentaron en las sub-escalas de Introvertido (7 niños) y Atención (5 niños) (figura 1), 
Tabla 3. Percentiles del Cuestionario por grupo de edades.

\begin{tabular}{|c|c|c|c|c|c|c|c|c|c|c|c|}
\hline \multicolumn{12}{|c|}{4 años (entre 46 y 57 meses) } \\
\hline & I & II & III & IV & $\mathbf{V}$ & VI & VII & Otros & I & $\mathbf{E}$ & G \\
\hline$\leq 84$ & 4,00 & 3,00 & 4,00 & 2,00 & 4,00 & 3,00 & 7,00 & 5,00 & 12,00 & 10,00 & 9,00 \\
\hline 93 & 5,00 & 4,00 & 5,00 & 3,00 & 5,00 & 4,00 & 10,00 & 8,00 & 13,00 & 13,00 & 14,00 \\
\hline 98 & 6,00 & 4,00 & 7,00 & 4,00 & 6,00 & 5,00 & 13,00 & 23,00 & 17,00 & 18,00 & 26,00 \\
\hline$\geq 100$ & 7,00 & 5,00 & 8,00 & 5,00 & 7,00 & 6,00 & 14,00 & 24,00 & 18,00 & 19,00 & 27,00 \\
\hline \multicolumn{12}{|c|}{5 años (entre 58 y 69 meses) } \\
\hline$\leq 84$ & 5,00 & 3,00 & 3,00 & 2,00 & 3,00 & 3,00 & 8,00 & 5,00 & 13,00 & 11,00 & 8,00 \\
\hline 93 & 6,00 & 4,00 & 4,00 & 3,00 & 4,00 & 4,00 & 10,00 & 6,00 & 15,00 & 13,00 & 9,00 \\
\hline 98 & 7,00 & 5,00 & 5,00 & 4,00 & 5,00 & 5,00 & 13,00 & 10,00 & 16,00 & 17,00 & 14,00 \\
\hline$\geq 100$ & 8,00 & 6,00 & 6,00 & 5,00 & 6,00 & 6,00 & 14,00 & 11,00 & 17,00 & 18,00 & 15,00 \\
\hline \multicolumn{12}{|c|}{6 años (entre 70 y 78 meses) } \\
\hline$\leq 84$ & 5,00 & 4,00 & 4,00 & 2,00 & 4,00 & 4,00 & 9,00 & 5,00 & 14,00 & 12,00 & 10,00 \\
\hline 93 & 6,00 & 5,00 & 5,00 & 4,00 & 5,00 & 5,00 & 11,00 & 7,00 & 16,00 & 15,00 & 11,00 \\
\hline 98 & 7,00 & 6,00 & 6,00 & 6,00 & 6,00 & 6,00 & 13,00 & 10,00 & 20,00 & 18,00 & 15,00 \\
\hline$\geq 100$ & 8,00 & 7,00 & 7,00 & 7,00 & 7,00 & 7,00 & 14,00 & 11,00 & 21,00 & 19,00 & 16,00 \\
\hline
\end{tabular}

Nota: I Emocionalmente Reactivo, II Ansiedad - Depresivo, III Quejas somáticas, IV Introvertido, V Problemas del Sueño, VI Problemas Atención, VII Agresivo, Otros problemas, I Internalizante, E Externalizante y G Problemas Generales. Escala Típica (percentiles $\leq 84$ ), Escala Preclínica (percentiles entre 93 y 98) y Escala Clínica (percentiles $\geq 100$ ).

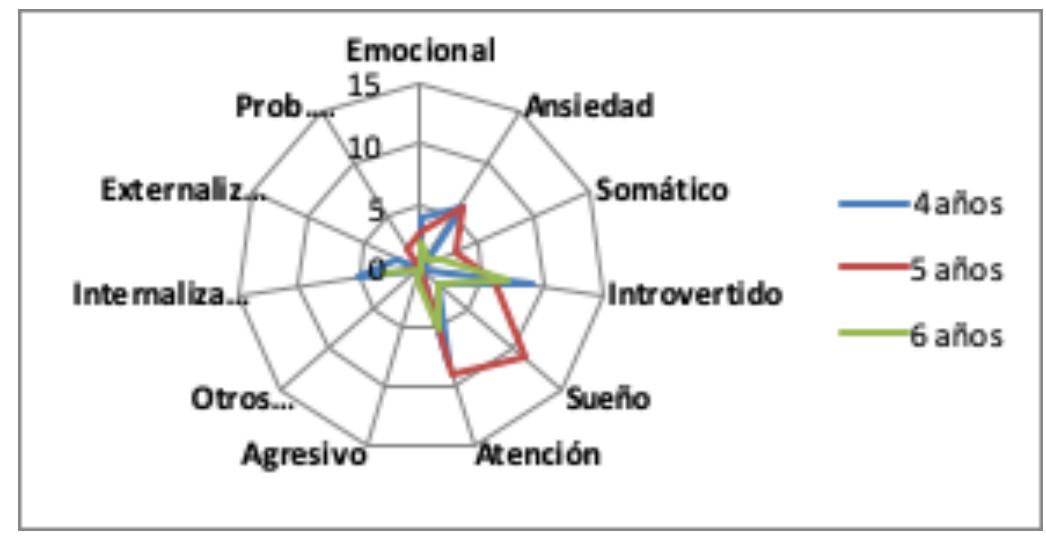

Figura 1. Cantidad de niños de N2 con rasgos preclínicos.

mientras que 1 niño tuvo rasgos clínico de introvertido (figura 2).

\section{Validez de contenido}

De 99 ítems analizados, se reportaron 97 ítems con buena y aceptable discriminación (96,7\%). Mientras que 2 ítems (3,03\%) mostraron mala discriminación. Las sub-escalas que tuvieron ítems con mala discriminación fueron: Introvertido (ítems (70) y
Otros problemas (ítems (3) como se muestra en la tabla 4.

Con respecto al índice de dificultad, el 74,7\% de los ítems (74 ítems) mostraron índices de dificultad alta (difíciles), el 17,1\% de los ítems (17 ítems) mostraron índice de dificultad media (ni fácil, ni difícil) y el 9,09\% (9 ítems) mostraron índices de dificultad bajo (fáciles) como se muestra en la tabla 4. 


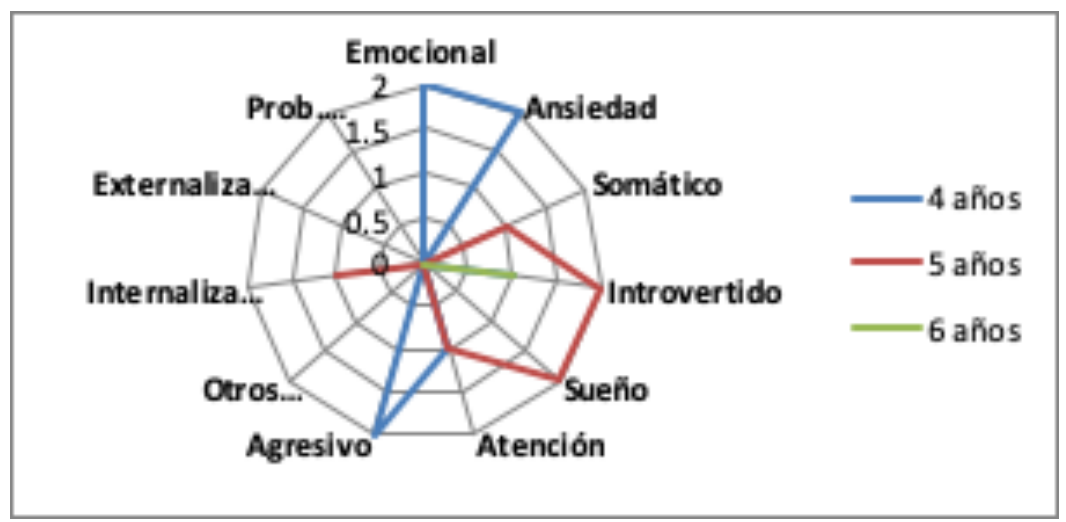

Figura 2. Cantidad de niños de N2 con rasgos clínicos.

Tabla 4. Análisis de ítems del cuestionario.

\begin{tabular}{|c|c|c|c|}
\hline \multirow[b]{2}{*}{ Índice de Dificultad } & \multicolumn{3}{|c|}{ Índice de Discriminación (correlación ítem-total) } \\
\hline & Buena $(\geq, 20)$ & $\begin{array}{l}\text { Aceptables } \\
(\mathrm{e} /, 10 \mathrm{y}, 19)\end{array}$ & $\begin{array}{l}\text { Mala } \\
(<, 10)\end{array}$ \\
\hline $\begin{array}{l}\text { Baja } \\
(\geq, 80)\end{array}$ & $90,1,12,52,67$ & $50,55,73,91$ & \\
\hline $\begin{array}{l}\text { Media } \\
(\mathrm{e} /, 51 \mathrm{y}, 79)\end{array}$ & $10,22,5,71,98,69,3,11,14,17$ & $79,30,32,34,36,49,97$ & \\
\hline $\begin{array}{l}\text { Alta } \\
(\leq, 50)\end{array}$ & $\begin{array}{c}82,83,68,78,74,59,95,18,20,44,58 \\
66,46,37,47,39,4,48,6,56,8,15,16 \\
29,81,96,51,92,99,33,87,7,19,24 \\
45,86,64,84,94,27,35,42,85,9,31 \\
41,54,57,60,61,63,65,72,75,76,77 \\
80,88,89\end{array}$ & $\begin{array}{c}40,53,, 13,25,26,28,49 \\
, 43,93,2,23,62,38\end{array}$ & 21,70 \\
\hline $\begin{array}{l}\text { Nota: Emocionalmente I } \\
33,37,43,47,68,87 \mathrm{y} \\
\text { Introvertido (ítems } 2,4, \\
\text { escala 6: Problemas de a } \\
40,42,44,53,58,66,69 \\
34,36,41,49,50,54,55\end{array}$ & $\begin{array}{l}\text { activo (ítems 21, 46, 51, 79, 82, 83, 92, } 97 \text { y 99) } \\
\text { ). Sub-escala 3: Quejas somáticas (ítems } 1,7,12 \\
\text { 62, 67, 70, 71 y 98). Sub-escala 5: Problemas en } \\
\text { nción (ítems 5, 6, 56, 59, 95). Sub-escala 7: Cond } \\
81,85,88 \text { y 96). Sub- escala 8: Otros problemas } \\
57,60,61,63,65,72,73,75,76,77,80,89,91 \text { ) }\end{array}$ & $\begin{array}{l}\text { Sub-escala 2: Ansiedad - Depre } \\
19,24,39,45,52,78,86 \text { y } 93 \text { ) } \\
\text { l sueño (ítems } 22,38,48,64,74 \\
\text { cta agresiva (ítems } 8,15,16,18 \text {, } \\
\text { tems } 3,9,11,13,14,17,25,26 \text {, }\end{array}$ & $\begin{array}{l}\text { (ítems } 10 \text {, } \\
\text { - escala } 4: \\
\text { y } 94 \text { ). Sub- } \\
27,29,35 \text {, } \\
30,31,32 \text {, }\end{array}$ \\
\hline
\end{tabular}

La investigación utilizó la traducción al castellano de los argentinos Vázquez y Samaniego (6), por tanto fue necesario una revisión por expertos. El análisis indicó algunas modificaciones en diversos ítems en función de una mejor compresión por los padres. Se modificó el ítem 9 "Mastica lo que no es comestible" por "Mastica o Muerde lo que no es comestible, Ej. La manga de la camisa". El ítem 31 "Come o bebe cosas que no son comida" por "Come o bebe cosas que no son alimentos (no incluya dulces)". El ítem 35 "Se mete en muchas peleas" por "Pelea mucho o se mete en muchas peleas". El ítem 41 "Aguanta la respiración" por "Aguanta la respiración cuando se pone bravo o desea algo". El ítem 45 "Nauseas" por "Náuseas (deseo de vomitar), se siente mal". El ítem 56 "Es torpe" por "Es torpe y tosco". El ítem 67 "No responde al afecto" por "No responde al afecto o caricias mostradas por los adultos u otros niños". El ítem 79 "Súbitos cambios de tristeza a excitación" por "Cambios bruscos de tristeza a excitación". El ítem 82 "Súbitos cambios de humor o sentimientos" por "Es malhumorado". El ítem 83 "Se pone de malhumor a menudo" por "Se pone furioso con frecuencia". 


\section{Validez de estructura interna}

Según la prueba KMO, el nuevo instrumento puede someterse a un análisis de factores, pues mostró buenos valores $(0,84)$, por encima de 0,75 . De esta forma, el análisis de factor mostró que las 8 sub-escalas se agrupan en 3 factores. El factor 1 Externalizante con las sub-escalas Problemas de Atención, Conductas Agresivas, Problemas de Sueño y Otros problemas explican el $34,79 \%$ de la varianza del cuestionario. El factor 2 Internalizante, explica el $33,27 \%$ de la varianza y agrupa las sub-escalas Quejas somáticas, Emocionalmente reactivo, Ansiedad Depresivo y Otros problemas. Finalmente, el factor 3 Introvertido, agrupa la sub-escala Introvertido para explicar el $13,67 \%$ de la varianza del cuestionario. Los 3 factores explican el $81,74 \%$ de la varianza total del cuestionario.

\section{Confiabilidad}

La confiabilidad del cuestionario a través del método alfa cuando el ítem es eliminado mostró valores entre 0,84 y 0,85 . El análisis de correlación de ítem - total indicó que el 96,7\% de los ítems mostraron correlaciones adecuadas y buenas, mientras que solo 2 ítems mostraron valores de baja correlación (ítems 21 y 70$)$.

\section{DISCUSIÓN}

\section{El Cuestionario Child Behavior Checklist} (CBCL) mostró adecuados resultados de validación y estandarización en la población preescolar cubana. Este resultado responde a la necesidad de contar con pruebas válidas en la población preescolar cubana para evaluar el desarrollo socio afectivo y la frecuencia en que se expresan las conductas negativas en estas edades.

La adaptación lingüística y cultural del cuestionario fue adecuada y se pudo constatar en la cantidad de ítems que fueron respondidos por los padres con buena y aceptable discriminación (96,7\% de los ítems). Además los ítems fueron escrito con un lenguaje fácil y compresible para los padres, tal y como se muestra en los 10 ítems que se modificaron (análisis de expertos). Con igual propósito, es importante destacar que el $96,7 \%$ de los ítems mostró una correlación ítem - total con valores adecuados, lo cual indica que la mayoría de los ítems del cuestionario contribuyeron a explicar la varianza de la prueba y por consiguiente a explicar las sub-escalas que describe el cuestionario.
De esta forma, el análisis de validez de contenido y la confiabilidad han mostrado valores estadísticos apropiados para un análisis inicial del cuestionario en la población preescolar de Cienfuegos. No obstante, es necesario realizar un segundo estudio donde se aumente la muestra para obtener una mayor variabilidad y ajuste de las normas o percentiles.

A pesar de la necesidad de realizar un segundo estudio en una muestra nacional, los percentiles obtenidos permitieron clasificar el $88,3 \%$ de los niños con alteraciones en la muestra $\mathrm{N} 2$, específicamente por introversión, problemas de atención, problemas de sueño y ansiedad. Es importante destacar que la mayoría de los niños con alteraciones socio afectiva fueron varones $(75 \%)$, lo cual es consistente con varias investigaciones donde expresan la preocupación del desarrollo cognitivo y emocional en el sexo masculino $(14,15,16)$. Desde esta mirada, se sugiere realizar un análisis más crítico y profundo respeto al desarrollo de la población masculina, pues son más sensibles a presentar alteraciones neuropsicológicas y emocionales $(17,18)$.

En específico, los autores de este artículo destacan la importancia de evaluar con profundidad al niño de sexo masculino con alteraciones en el neurodesarrollo, tanto con expresiones exteriorizantes de la conducta (hiperactividad, agresividad, problemas de atención) como los niños con manifestaciones interiorizantes (miedos, ansiedad, problemas de sueño, depresión). Es común encontrar en la consulta una alta cantidad de niños con manifestaciones exteriorizantes, ya que es una "molestia" para el educador y para los padres, sin embargo, los niños con expresiones emocionales de tipo interiorizantes no tiene la misma preocupación familiar debido a su expresión silente y de poca molestia para los demás. . Sin dudas, es una preocupación de talla internacional, pues varios autores $(1,2,3)$ reconocen el impacto negativo que tienen los problemas emocionales de naturaleza internalizantes en la futura salud mental del niño. La presente investigación se suma a esa preocupación, ya que la mayoría de los niños evaluados en N2 presentan más expresiones emocionales de tipo interiorizantes (Ansiedad, Introvertido, Sueño) que exteriorizante (Atención y Agresividad).

En estudios anteriores realizados por los autores $(19,20)$, se había demostrado que los niños con alteraciones en su neurodesarrollo son proclives a presentar hiperactividad o agresividad o ambos en comorbilidad, sin embargo los resultados obtenidos han 
revelado que acompañado de esos síntomas también ocurren las conductas internalizantes (problemas de sueño, ansiedad, introvertido, somatizaciones). De este argumento se desprende la necesidad de aplicar el cuestionario CBLC en la población preescolar por la variedad de síntomas que se pueden detectar y tratar a tiempo.

El análisis confirmatorio de factores indicó que el cuestionario puede clasificar al niño a través de dos factores: Interiorizante $(33,27 \%$ de la varianza) y el Exteriorizante $(34,79 \%$ de la varianza), los cuales explican la mayor parte de la varianza del instrumento. No obstante, surge como tercer factor la Introversión, por tanto se podría revisar este resultado en otras investigaciones, pues al parecer en la muestra N1 habían varios padres que destacaron este rasgo en el niño. Un segundo estudio debe ir orientado a subsanar algunas limitaciones metodologías, tales como el ajuste de los percentiles, el análisis de factores y la correlación del cuestionario con otros instrumentos.

En conclusiones, el cuestionario CBCL versión para padres es una herramienta para detectar tempranamente niños entre 4 y 6 años con alteraciones en su desarrollo socio afectivo en la población de Cienfuegos. Ofrece dos resultados de vital importancia para el profesional, síntomas con rasgo preclínico, los cuales son afectaciones emocionales ligeras y moderadamente intensas en la expresión conductual del niño, pero de obligado seguimiento y tratamiento educativo, y los síntomas con rasgos clínicos, considerados intensos, persistentes y con tratamiento farmacológico y educativo. El profesional cubano puede usar los percentiles que ofrece el artículo, aunque debe usarlos con cierta precaución, ya que el estudio ha sido realizado solo en una provincia del país. No obstante, el cuestionario se está aplicando en cuatro provincias en el país con fines de elaborar una normativa nacional.

\section{Correspondencia}

Yaser Ramírez-Benítez

Correo electrónico: yramirezbenitez@gmail.com

\section{REFERENCIAS BIBLIOGRÁFICAS}

1. Stülb K, Messerli-Bürgy N, Kakebeeke TH, Arhab A, Zysset AE, Leeger-Aschmann CS, et al. Prevalence and predictors of behavioral problems in healthy Swiss preschool children over a one-year period. Child Psych Hum D. 2019; 50(3); 439-448.
2. Rescorla LA, Genaro B, Ivanova MY, International ASEBA Consortium. International comparisons of emotionally reactive problems in preschoolers: CBCL $/ 1 \frac{1}{2}-5$ Findings from 21 Societies. J Clin Child Adolesc. 2019; 28:1-14.

3. Lohndorf RT, Vermeer HJ, Cárcamo RA, De la Harpe C, Mesman J. Preschoolers' problem behavior, prosocial behavior, and language ability in a LatinAmerican context: The roles of child executive functions and socialization environments. Early Child Res Q. 2019; 48: 36-49.

4. Rescorla L, Achenbach M, Ivanova Y, et al. International comparisons of behavioral and emotional problems in preschool children: Parents' reports from 24 societies. J Clin Child Adolesc. 2011; 40(3): 456-467.

5. Reynolds R, Kamphaus W. BASC: Sistema de evaluación de la conducta en niños y adolescentes. Madrid: TEA; 2004.

6. Lecannelier F, Pérez JC, Groissman S, et al. Validación del inventario de conductas infantiles para niños de entre $1 \frac{1}{2}-5$ años (CBCL $1 \frac{1}{2}-5$ ) en la Ciudad de Santiago de Chile. Univ Psychol. 2014; 13(2): 491500 .

7. Montiel-Nava C, Montiel-Barbero I, Peña JA. Datos normativos del cuestionario sobre el comportamiento de niños versión para padres (CBCL) y versión para profesores (TRF) en una muestra de niños venezolanos. Psicol Conduct. 2007; 15 (1): 29-44.

8. Vázquez N, Samaniego C. Estandarización del Child Behavior Checklist para preescolares de población urbana de Argentina. Revista Evaluar. 2017; 17(1): 65-79.

9. Albores-Gallo L. Consistencia interna y validez de criterio de la versión mexicana del Child Behavior Checklist 1.5-5 (CBCL/1.5-5). Rev Chil Pediatr. 2016; 87(6): 455-462. Doi: 10.1016/j.rchipe.2016. 05.009

10. Ramírez Y, Bernal F. Prevalencia de alteraciones cognitivas en la provincia Cienfuegos. Revista Cubana de Pediatría. 2020; 92 (3):1-18.

11. Backhoff E, Larrazolo N, Rosas M. The level of difficulty and discrimination power of the basic knowledge and skills examination. Revista Electrónica de Investigación Educativa. 2000; 2 (1): 1-16.

12. Crocker L, Algina J. An introduction to classical and modern test theory. New York: Holt, Rinehart \& Winston; 1986.

13. Diaz-PortilloJ.Guíaprácticadelcursodebioestadística aplicada a las ciencias de la salud. Madrid: Instituto Nacional de Gestión Sanitaria; 2011.(Fecha de acceso: 15 de julio del 2020) Disponible en: https://www.academia.edu/10906749/ Gu\%C3\%ADa_Pr\%C3\%A1ctica_del_Curso_de Bioestad\%C3\%ADstica_Aplicada_a_las_Ciencias_ 
de_la_Salud

14. Haas LM, McArthur BA, Burke TA, Olino TM, Abramson LY, Alloy LB. Emotional clarity development and psychosocial outcomes during adolescence. Emotion. 2019; 19(4): 563-572. Doi: 10.1037/emo0000452

15. Lara LM, Bos MG, Van Rossenberg F, Crone EA. Sex effects on development of brain structure and executive functions: Greater variance than mean effects. J Cognitive Neurosci. 2019: 31 (5): 730-753.

16. Todd RM, Miskovic V, Chikazoe J, Anderson AK. Emotional objectivity: Neural representations of emotions and their Interaction with cognition. Annu Rev Psychol. 2020; 71:25-48. Doi: 10.1146/ annurev-psych-010419-051044

17. Muñoz-Suazo MD, Navarro-Muñoz J, Díaz-Román A, Porcel-Gálvez AM, Gil-García E. Sex differences in neuropsychological functioning among children with attention-deficit/hyperactivity disorder. Psychiatry Res. 2019; 278: 289-293.
18. Kohls G, Baumann S, Gundlach M, Scharke W, Bernhard A, Martinelli A, et al. Investigating sex differences in emotion recognition, learning, and regulation among youths with conduct disorder. J Am Acad Child Adolesc Psychiatry. 2020; 59(2): 263273.

19. Ramírez Y, Jiménez-Morales RM, Díaz M, Fárdales $\mathrm{V}$. Normas cubanas del instrumento neuropsicológico Luria Inicial para niños preescolares 4-6 años. Cuadernos de Neuropsicología. 2015; 9 (2): 49-69.

20. Ramírez Y, Jiménez RM. Aplicación de la prueba Luria Inicial en el niño preescolar con desviaciones del neurodesarrollo en Cuba. Gac Med Espirit. 2014; 16 (3): 1-9. (Fecha de acceso: 15 de julio del 2020) Disponible en: http://scielo.sld.cu/scielo.php? script $=$ sci_arttext\&pid $=$ S1608-89212014000300019 $\& \operatorname{lng}=$ es\&nrm $=$ iso

Recibido: 26/08/2019 Aceptado: 21/09/2020 